\title{
Individuals with Autism: Analysis of the First Interaction with Nao Robot Based on Their Proprioceptive and Kine....
}

\section{Chapter · August 2015}

DOI: 10.1007/978-3-319-21290-6_23

\section{CITATIONS}

6

4 authors:

\section{Pauline Chevalier}

University of Twente

13 PUBLICATIONS 25 CITATIONS

SEE PROFILE

\section{Jean-Claude Martin}

Computer Sciences Laboratory for Mechanics. . 225 PUBLICATIONS $\quad 2,398$ CITATIONS

SEE PROFILE
READS

179
Brice Isableu

Aix-Marseille Université

75 PUBLICATIONS 1,011 CITATIONS

SEE PROFILE

Adriana Tapus

MINES ParisTech

95 PUBLICATIONS 1,713 CITATIONS

SEE PROFILE

Some of the authors of this publication are also working on these related projects: 


\title{
Individuals with Autism: Analysis of the First Interaction with Nao Robot based on their Proprioceptive and Kinematic Profiles
}

\author{
Pauline Chevalier ${ }^{1}$, Brice Isableu ${ }^{2}$, Jean-Claude Martin $^{3}$ and Adriana Tapus ${ }^{1}$ \\ ${ }^{1}$ Robotics and Computer Vision Lab, ENSTA-ParisTech, Palaiseau, 91120, France \\ \{pauline.chevalier; adriana.tapus\} @ensta-paristech.fr \\ ${ }^{2}$ CIAMS-Motor Control and Perception, Univ Paris Sud, Orsay, 91405, France \\ brice.isableu@u-psud. fr \\ ${ }^{3}$ Cognition, Perception, Use, LIMSI-CNRS, Orsay, 91403, France \\ martinelimsi.fr
}

\begin{abstract}
Our research aims to develop a new personalized social interaction model between a humanoid robot and an individual suffering of Autistic Spectrum Disorder (ASD), so as to enhance his/her social and communication skills. In order to define individual's profile, we posit that the individual's reliance to proprioceptive and kinematic visual cues will affect the way an individual suffering of ASD interacts with a social agent. We describe a first experiment that defines each participant's perceptive-cognitive and sensorimotor profile with respect to the integration of visual inputs, thanks to the Sensory Profile questionnaire and an experimental set-up. We succeeded to form 3 groups with significant different behavioural responses inside our subject pool formed by 7 adults and 6 children with ASD. In a second experiment, we presented the Nao robot to all of our participants. We video-analysed their behaviours and compared them to the profiles we defined. In view of our results, this first interaction confirmed our hypothesis: participants with a weak proprioceptive integration and strong visual dependency had more successful interaction than participants with an overreliance on proprioceptive input and hypo-reactivity to visual cues.
\end{abstract}

Keywords: Autism; Personalized Interaction; Socially Assistive Robotics; Proprioception; Kinematics

\section{$1 \quad$ Introduction}

Nowadays, research in socially assistive robotics (SAR) is in expansion [1,2]. One of the target populations is people suffering of Autistic Spectrum Disorders (ASD). Individuals with ASD have impaired skills in communication, interaction, emotion recognition, joint attention, and imitation [3]. Many studies showed that children with ASD have a great affinity with robots, computers, and mechanical components [4]. Moreover, in SAR, robots have already been used several times as tools in socialization therapies for children with ASD [5, 6]. Individuals with ASD also suffer from 
visual and sensory motor impairments [7, 8]. It is suggested that they manifest an exacerbated reliance on proprioceptive feedback and a deficient use of kinematic visual cues $[9,10]$. A link between the individual integration of proprioceptive and visual feedbacks and communication, interactions skills, and emotion recognition had already been discussed in [10].

The goal of our research is to develop a novel personalized robot behaviour based on a social interaction model for individuals suffering of ASD. In order to define users' profiles, we make the hypothesis $\mathrm{H} 1$ - that mitigated behavioural response (i.e., hypo reactivity) to visual motion of the scene and overreliance on proprioceptive information are linked in individuals with ASD to difficulties in integrating social cues and engaging in successful interactions. We work in collaboration with two care facilities for people suffering of ASD: MAIA Autisme (France), an association for children and adolescents with ASD, and FAM-La Lendemaine (France), a residence for adults with ASD. Our current subject pool is composed of 6 autistic children $(10.9 \pm 1.8$ years) and 7 autistic adults (26.1 \pm 7.9 years) from these two care facilities. Both of the experiments described here have been done with the same individuals ${ }^{1}$.

In this paper, we first present an experiment that defines each participant's perceptive-cognitive and sensorimotor profiles with respect to the integration of visual inputs. Furthermore, we describe a second experiment where the Nao robot was presented for the first time to all our participants. Their behaviours during this first interaction with the robot, based on their profiles, are analysed and reported.

\section{State of the art}

Motor, sensory, and visual impairments are present in autism, but are not taken into account in the ASD diagnosis [7, 8]. These deficits have an influence on the quality of life of individuals suffering of ASD and on their social development. An overreliance on proprioceptive information in autism has already been studied. As it has been reported in [8], individuals with autism showed normal to improved integration of the proprioceptive cues in comparison to typically developed individuals. Moreover, results in [9] confirmed that postural hypo reactivity to visual information was present in the tested individuals with autism (individuals suffering from ASD with IQ comparable to that of typically developed persons). Proprioception integration in ASD was also studied so as to better understand the interaction and social capabilities. In [10], Haswell and al. asked 14 children with ASD and 13 typically developed children to perform a reaching task with their arm, while holding a robotic arm that applied a force, constraining the movement. It was found that autistic brain built a stronger than normal association between self-generated motor commands and proprioceptive feedback, confirming an overreliance on proprioceptive cues in individuals with ASD. Furthermore, it was also established that greater the reliance on proprioception was, the higher the impairments in social and imitation functions were.

1 For confidentiality reasons, we encoded the participants' identity: AD\# for the participants from the adults care centre and $\mathrm{CH} \#$ for the participants from the children care centre. 
The use of robots in therapy for individuals with ASD has been a great topic of interest in the last decade. Indeed, robots have been found to be great partners in learning social interaction, and imitation skills [4]. Robots are less complex in their behaviour than human peers offering more predictable and comfortable interaction behaviours [6].Different research areas on robots for therapy for individuals with ASD have been reported in [1]: (1) developing a robot especially designed for individuals suffering of ASD, (2) design of Human-Robot Interaction, and (3) evaluation of the robot in therapies. There are many open challenges in SAR for people with ASD. However, due to small subject pools and/or short-time experiments, generalized results in improved skills are still questioned [11]. Salter et al. [12] discussed on the variability of Human-Robot Interaction set up that could biased the results and founding in this area. To the best of our knowledge, no study examined the sensorimotor profiles (visual dependency) of individuals suffering of ASD to determine and elaborate individualized and personalized scenarios for Human-Robot social interaction therapy.

\section{Defining proprioceptive and kinematic profiles}

\subsection{Methods}

The first step of our work was to determine how to define each participant's perceptive-cognitive and sensorimotor profiles. We used two methodologies. First, the Sensory Profile (SP) [13] and the Adolescents/Adults Sensory Profiles (AASP) [14] were filled. They allowed evaluating individual's sensory processing preferences, being widely used in ASD research. The individual's sensory processing preferences are described in terms of the quadrants in Dunn's Model of Sensory Processing [13]: Low Registration, Sensation Seeking, Sensory Sensitivity, and Sensation Avoiding. These quadrants are formed by the junctions between the individual differences in neurological thresholds for stimulation (high-low) and self-regulation strategies (activepassive).

As we worked with two age groups, we used the appropriate questionnaire corresponding to their age range. The AASP was filled for all the +11 years old participants and the SP was filled for the youngest participants. As most of the participants did not have the cognitive level to fill the questionnaire by themselves, it has been filled with the help of their caretakers who knew well their habits and response to everyday life sensory stimuli. The caretakers were well informed of the conditions and forms of the questionnaire. In order to have homogeneous scores between age groups to assess Movement, Visual, Touch, and Auditory processing, we carefully matched and adapted the items based on their correspondence to the neurological threshold and behaviour response/self-regulation. Second, we designed an experimental setup to assess the effect of a moving virtual visual scene (VVS) on postural control, and the individual's capability to use proprioceptive inputs provided in dynamics of balance to reduce visual dependency [14]. In an instable posture, the integration of proprioceptive feedback differs among individuals. An individual integrating proprioceptive cues less than other individuals is probability visual dependent. 


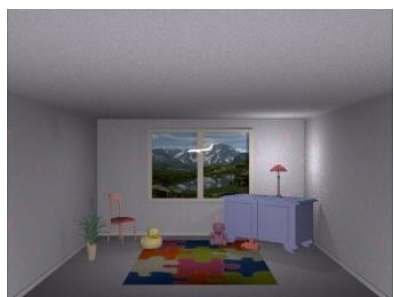

(b)

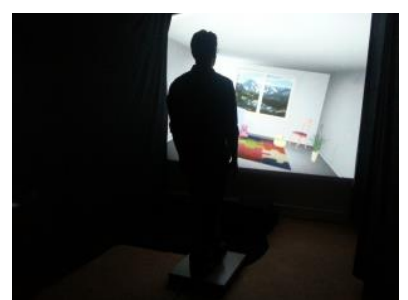

(a)

Fig.1. Setup: (a) Capture of the virtual room used in the experiment; (b) Experimental setup for the adults group in condition $\mathrm{C} 3$

While exposed to a visual motion in an instable posture, his/her body sway will follow the visual stimulus. Participants were asked to stand on a force platform (FP) in front of a virtual room (Fig. 1) in three conditions:

- C1 - Stable position with static VVS: each participant stood on the FP, straight ahead, with feet hip-width apart. The virtual room stays still. The recording lasted 30 seconds.

- C2 - Stable position with moving VVS: the participant stood on the FP, straight ahead with feet hip-width apart. The virtual room has a sinusoidal movement. The recording lasted 50 seconds.

- C3 - Tandem Romberg position with moving VVS: the participant stood on the FP, straight ahead, one foot in front of the other one (tandem Romberg stance). The virtual room has a sinusoidal movement. The recording lasted 50 seconds.

The virtual room was created with Blender, a free 3D rendering software. It was decorated with child toys and furniture so as to create a friendly environment. A toy plane was placed in the line of sight of the individuals in order to help them to focus on the task, and not to be distracted away. It was projected to a wall with a short focal projector in a dark room. It rolled at $0.25 \mathrm{~Hz}$ with an inclination of $\pm 10^{\circ}$. For the adult group setup, the dimension of the projection was $2.4 \mathrm{~m}$ large $\mathrm{x} 1.8 \mathrm{~m}$ high and the participants stood at $1.3 \mathrm{~m}$ of the point of observation (Fig.1b). For the children group setup, the dimension was $1.75 \mathrm{~m}$ large $\mathrm{x} 1.16 \mathrm{~m}$ high and the participants stood at $1 \mathrm{~m}$.

\subsection{Data Analysis}

For all the sessions, a FP (AMTI OR6-5-1000) was used to record the displacement of the centre of pressure $(\mathrm{CoP})$ with a sampling frequency of $1 \mathrm{KHz}$. We used a Butterworth filter with a cut-off frequency of $10 \mathrm{~Hz}$ on the recorded data in order to reduce the noise. We computed the Root Mean Square (RMS) of the CoP in both mediolateral and anteroposterior directions as an indicator of the individual's stability. Indeed, the RMS provides the information about the variability of the CoP in space [15]. We computed the Fast Fourier Transform (FFT) of the CoP and the correlation with a $0.25 \mathrm{~Hz}$ sinus so as to evaluate coupling between the postural response and the moving visual stimulus. In order to assess the correlation between sensory preferences and postural responses to our visual stimulus, we computed the correlation between the scores of the different items of the SP and the data obtained from the Cop. We comp- 

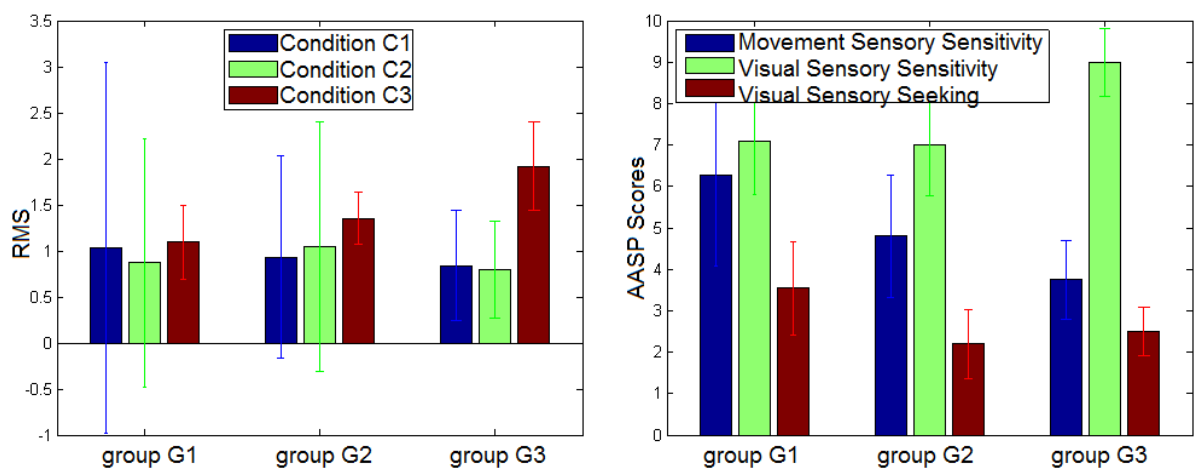

Fig.2. Left: Histograms of the mean RMS for the groups defined by clustering analysis for the 3 conditions; Right: Histograms for AASP scores for the groups.

uted the correlation between the CoP behaviours during different conditions to evaluate if a behaviour in a condition induced another behaviour in a different one. Then, we used clustering analysis (Dendrogram) to form behavioural groups.

\subsection{Results}

We observed a close relationship between the AASP patterns and the postural behaviours. Individual with higher movement sensory sensitivity score in AASP showed greater postural stability, and postural sway less driven by the moving VVS. A low visual sensation seeking behaviour also resulted in smaller postural responses to the moving VVS whereas individual with a higher visual sensitivity showed a greater postural coupling response with moving VVS. A relation between age and postural instability was found, but not between age and postural response to the moving VVS.

Clustering analyses allowed us to identify 3 groups with significant different behavioural responses (see Fig. 2): (G1) high scores in movement sensitivity and visual sensation seeking, low scores in visual sensory sensitivity and strong visual independence to the moving VVS, suggesting an overreliance on proprioceptive input and hypo reactivity to visual cues; (G2) moderate scores in movement sensitivity and low scores in visual sensation seeking, low scores in visual sensory sensitivity and moderate reactivity to moving VVS, suggesting a reliance on both visual and proprioceptive input; and (G3) high scores in visual sensory sensitivity and low scores in movement sensitivity and in visual sensory seeking, and hyper reactivity to moving VVS, suggesting a weak proprioceptive integration and strong visual dependency.

\section{Greetings with Nao}

\subsection{Method}

A first Human-Robot Interaction was conducted with all our participants. The interaction purpose was to present Nao to our participants for a short duration, up to 2 minutes. Indeed, some of the individuals with ASD are reluctant to unusual events 
and change in their daily routine. The scenario was the following: after being seated in front of Nao, the robot waved to the participant and said "Hello, I am Nao. You and I, are going to be friends". If the participant was verbal, the robot asked for the participant's name. Afterwards, it continued with "Hello" followed by the name of the participant, and asked if he/she wanted it to dance for him/her, and then danced. During all the experiment, the participant was with his/her caretaker. The caretaker encouraged the participant to look at and answer to the robot. We manually analysed the video recording the participants' gaze direction and gestures towards the robot, the caretaker, and all the other directions so as to determine a match with the 3 groups described in section 3.3, so as to evaluate our H1 hypothesis (Table 1).

Table 1. Mean percentage of the gaze direction for each group

\begin{tabular}{|l|l|l|l|}
\hline Gaze direction & G1 & G2 & G3 \\
\hline Toward the robot & $62,58 \%$ & $69.06 \%$ & $89.19 \%$ \\
\hline Toward the caretaker & $13,84 \%$ & $1.49 \%$ & $1.59 \%$ \\
\hline Other & $23.57 \%$ & $29.45 \%$ & $9.21 \%$ \\
\hline
\end{tabular}

\subsection{Results}

The analysis of the gaze direction showed us that the individuals from G3 had gaze direction more focused on Nao and switched less their gaze than the individuals belonging to other groups. The individuals from G1 were less looking at Nao, they switched more their gaze, and looked more in other directions (nor the robot or the caretaker) than the other groups, see Table 1. Only two individuals (AD3 from G2 and AD6 from G1) looked less than 50\% of the time in other direction. The gesture analysis showed that individuals from $\mathrm{G} 3$ (two out of four: $\mathrm{CH} 2$ and $\mathrm{CH} 6$ ) had more social gestures than the other groups toward Nao robot, such as waving back to Nao while it was presenting itself. Only one of the individuals from G2 (AD1) had this behaviour and none of them was in G1. Two individuals (CH5, non-verbal, and CH6) took the initiative to touch the robot during the interaction. Overall, they were impressed by the machine. Participant AD1 recoiled when the robot moved its arms in her direction but she was the only one from G2 to wave back. The individuals from G3 showed more initiative to answer back to the robot. All of them answered to the robot after it asked the name and/or if he/she wanted it to dance. Half of the individuals from G2 had this same behaviour, and only one individual out of 4 of the G1 (CH5 from G1 is non-verbal so she is not taken into account). Moreover, participant AD4 from $\mathrm{G} 1$, who answered back, was really happy to interact with the robot.

\section{$5 \quad$ Conclusion and Discussions}

Thanks to our first experiment, we succeeded to form three groups between our participants, describing each participant's response to visual and proprioceptive inputs. 
With these results and our hypothesis $\mathrm{H} 1$, we were able to make assumptions on the behaviours each individual will have during the Human-Robot Interaction sessions. We posit from $\mathrm{H} 1$ that individuals from $\mathrm{G} 1$ will have less successful interaction than the ones from G2 and G3, and that individuals from G3 will have the most successful interactions. The interaction between the participants and the robot we conducted permitted us to analyse their behaviour toward the robots. The analysis confirmed our hypothesis made on the groups. Participants from G3 showed longer gaze direction, more speech and social gesture initiatives toward Nao and participants from G1 showed less social behaviour than the 2 other groups.

As it have been already seen in SAR almost all of our participants, children and adults, showed great interest to their new mechanical companion. We confirmed our hypothesis on the formed groups on a short greeting interaction. Moreover, the interaction was relevant because greetings are an important stage of social interaction. Presenting the robot before starting the long-term personalized interaction seemed to be an important first step, as in ASD new events can be stressful and feared.

As in [8], we did not find a relationship between age and postural behaviours, differently to typically developed individuals [17]. The groups G1 and G2 are linked to the results found in these studies. The profiles we defined gave us a group formed by individuals with a weak proprioceptive integration and strong visual dependency (G3), which is in contradiction with earlier studies showing our statement that individual with autism have an overreliance on proprioceptive cues. However, Molloy and al. [18] found an impairment of the proprioception input in autism, and that children with ASD used more the visual cues to reduce sway and maintain balance. In [19], the authors found that unlikely to typically developed individual, individuals with ASD have an impaired proprioception development. Their sensory-motor signal appear to remain at the kinaesthetic stage of typically developed 3-4 years old children, and have to rely on visual inputs. They also conjectured that the impaired proprioception of physical micro-movements of the individuals with ASD impedes as well their visual perception of micro-movements in others during real time interactions, impairing their abilities to interact with people.

These results will help us to model the customized Human-Robot Interaction sessions and adapt the robot's behaviours as a function of the participants' profile. We plan on developing a parameterized robot behaviour that will adapt its gaze, head and body posture, gestures, speech and facial expressions [11]. The collaboration with two care facilities allows us to observe the long-term effect of a therapy with humanoid robots on 13 participants, innovative in SAR for individuals with ASD. Indeed, most studies are on few days, weeks or month with subject pools up to 3-4 participants.

\section{Acknowledgments}

This work is supported by IdF Doctoral Fellowship France 2013, HANDICAP theme. Many thanks to G. Lerigoleur and C. Bazile. We also thank the participants, their families and the caretakers for their participation and support. 


\section{References}

1. Feil-Seifer, D. and M.J. Mataric: Defining socially assistive robotics. In 9th International Conference on Rehabilitation Robotics, 465-468 IEEE (2005)

2. Tapus, A., Mataric, M. J. and B. Scassellati: The Grand Challenges in Socially Assistive Robotics. In: IEEE Robotics and Automation Magazine (RAM), Vol. 14 (1) (2008)

3. Charman, T., Swettenham, J., Baron-Cohen, S., Cox, A., Baird, G. and A. Drew: Infants with autism: an investigation of empathy, pretend play, joint attention, and imitation. In: Developmental psychology, Vol. 33 (5), 781 (1997)

4. Hart, M.: Autism/excel study. In: Proceedings of the 7th International ACM SIGACCESS Conference on Computers and Accessibility, 136-141, ACM (2005)

5. Tapus, A., Peca, A., Aly, A., Pop, C., Jisa, L., Pintea, S., Rusu, A. S. and D.O. David: Children with autism social engagement in interaction with Nao, an imitative robot-A series of single case experiments. In: Interaction studies, Vol. 13 (3), 315-347 (2012)

6. Kim, E. S., Berkovits, L. D., Bernier, E. P., Leyzberg, D., Shic, F., Paul, R. and B. Scassellati: Social robots as embedded reinforcers of social behavior in children with autism. In: Journal of autism and developmental disorders, Vol. 43 (5), 1038-1049 (2013)

7. Simmons, D. R., Robertson, A. E., McKay, L. S., Toal, E., McAleer, P. and F.E. Pollick: Vision in autism spectrum disorders. In: Vision Research, Vol. 49 (22), 2705-2739 (2009)

8. Gowen, E. and A. Hamilton: Motor abilities in autism: a review using a computational context. In: Journal of autism and developmental disorders, vol. 43 (2), 323-344 (2013)

9. Greffou, S., Bertone, A., Hahler, E. M., Hanssens, J. M., Mottron, L. and J. Faubert: Postural hypo-reactivity in autism is contingent on development and visual environment: a fully immersive virtual reality study. In: Journal of autism and developmental disorders, Vol. 42 (6), 961-970 (2012)

10. Haswell, C. C., Izawa, J., Dowell, L. R., Mostofsky, S. H. and R. Shadmehr: Representation of internal models of action in the autistic brain. In: Nature neuroscience, Vol. 12 (8), 970-972 (2009)

11. Scassellati, B., Admoni, H. and M. Mataric: Robots for use in autism research. In: Annual Review of Biomedical Engineering, Vol. 14, 275-294 (2012)

12. Salter, T., Michaud, F. and H. Larouche: How wild is wild? A taxonomy to characterize the 'wildness' of child-robot interaction. Int. J. of Social Robotics, Vol. 2(4), 405-415 (2010)

13. Dunn, W.: Sensory profile: User's manual, In: The Psychological Corporation (1999)

14. Brown, C. and W. Dunn: Adolescent-Adult Sensory Profile: User's Manual. In: Therapy Skill Builders (2002)

15. Isableu, B., Fourre, B., Vuillerme, N., Giraudet, G. and M.A. Amorim: Differential integration of visual and kinaesthetic signals to upright stance. In: Experimental brain research, Vol. 212 (1), 33-46 (2011)

16. Chiari, L., Rocchi, L. and A. Cappello: Stabilometric parameters are affected by anthropometry and foot placement. In: Clinical Biomechanics, Vol. 17 (9), 666-677 (2002)

17. Molloy, C. A., Dietrich, K. N. and A. Bhattacharya: Postural stability in children with autism spectrum disorder. In: J. of autism and developmental disorders, Vol. 33 (6) (2003)

18. Torres, E. B., Brincker, M., Isenhower, R. W., Yanovich, P., Stigler, K. A., Nurnberger, J. I., Metaxas. D. N. and J.V. José: Autism: the micro-movement perspective. In: Frontiers in integrative neuroscience, Vol. 7 (2013)

19. Baumberger, B., Isableu, B. and M. Flückiger: The visual control of stability in children and adults: postural readjustments in a ground optical flow. In: Experimental brain research, Vol. 159 (1), 33-46 (2004) 